\title{
Modeling of an Intelligent Electronic Medical Records System
}

\author{
Min $\mathbf{W u}^{*}$ \\ Department of Health Informatics and Administration, University of Wisconsin-Milwaukee, USA \\ *Corresponding author: Min Wu, Department of Health Informatics and Administration, University of \\ Wisconsin-Milwaukee, USA
}

\begin{tabular}{|c|c|}
\hline ARTICLE INFO & ABSTRACT \\
\hline Received: July 04, 2019 & This paper proposes a model for intelligent EMRs, which includes integrated user \\
\hline Published: 幽 July 12, 2019 & $\begin{array}{l}\text { interfaces and multiplayer data models. The new model of EMRs should provide more } \\
\text { interoperability and Artificial Intelligence (AIs) features. }\end{array}$ \\
\hline
\end{tabular}

Citation: Min Wu. Modeling of an Intelligent Electronic Medical Records System. Biomed J Sci \& Tech Res 19(4)2019. BJSTR. MS.ID.003326.

\section{Introduction}

The integration of Electronic Medical Records (EMRs) into the busy health care workflow is a challenge. An initial challenge is to map specific informatics applications given different medical scenarios. To design an innovative computer-based health record is a complex research question. One important research task is to accurately document medical data. Recent studies are exploring the feasibility of an integrated and intelligent EMRs for general medicine. New models with intuitive user interfaces and Artificial Intelligence (AI) features will lead to wider EMRs use and optimize medical decision-making and health care. This study proposes some ideas for an intelligent model for EMRs, which will integrate new $5 \mathrm{G}$ and $\mathrm{AI}$ techniques into clinically care.

\section{Current Models of EMRs}

Based on the attributes of clinical data, EMRs can be documented in different formats, such as, problem oriented, timeoriented, and source-oriented. Most Electronic Medical Records have been designed around the Problem Oriented Medical Record (POMR) format. The design and implementation issues of POMR have been extensively studied [1]. The design of time-oriented Electronic Medical Records was based on the results of research in temporal querying and reasoning in medical informatics [2]. A recent study was to model electronic discharge summaries as a temporal constraint satisfaction problem [3].

\section{Formulation Process}

This project began with an analysis of current medical records to determine how much information is included in the medical record. It is aimed at revealing essential ideas found in the medical record. Current methodologies and applications were explored for how to represent, store and process medical information. This led to an integrated model to improve the user acceptance.

\section{Model Description}

\section{Integrated User Interfaces}

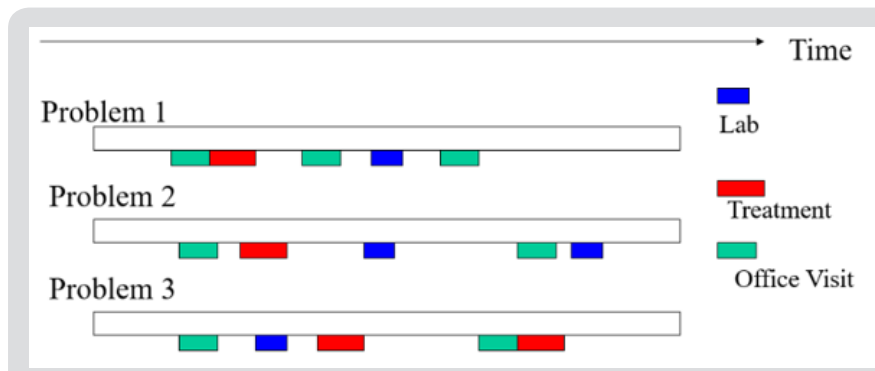

Figure 1: A user interface of the new EMRs.

Fast computing powers and 5G technologies can support new user interface design in EMRs. A user interface mockup in Figure 1 is proposed to help health care providers overview the whole patient records and understand complex workflows. The list of 
problems, locations and time features are integrated into one user interface. It can include multimedia records, such as, pictures, videos and radiographic findings.

\section{Multilayer Data Model}

A data model to support EMR, using multilayer concepts, is proposed to integrate the information in the different levels. It can include several layers, (1) spatial reference (patient body locations and shapes), (2) linked attributes and clinical information and (3) complex behaviors in medicine (procedures and guidelines).

\section{Interoperability}

The fifth generation of wireless networking technology (5G) enable us to connect many times more hospital devices to the network at once and remotely access at home. The new EMRs should aim to provide continuous monitoring of patients in certain diagnostic categories. For example, simultaneous measurement of heart rate will provide information about abnormalities of cardiovascular physiology at the time of a fall. It is believed that with these future developments, unobtrusive and wearable devices could advance health informatics and lead to fundamental changes of how healthcare is provided and reform the underfunded and overstretched healthcare systems.

\section{Artificial Intelligence (AI)}

The advancement of EMRs of using Artificial Intelligence in healthcare is a concept that has been investigated by many studies.
The machine learning technology can lead to predictions and make EMRs systems alive. For example, deep learning in combination with neuromorphic hardware can provide the basis for a wearable, real-time, always-on, patient-specific seizure warning system. As machine learning algorithms are increasingly used to support clinical decision making, it is vital to reliably quantify their prediction accuracy.

\section{Discussion and Conclusion}

Existing medical documentation formats do not precisely record medical information, because clinical observation is the primary data source. Despite of the availability of digital images and videos, multimedia information is not normally graphically annotated and integrated with other documentation in the medical record. This model proposes that clinical observation and multimedia data can be integrated to form a multilayer data model. The integrated and intelligent model is a challenge for medical informatics researchers, but a prototype system can be developed through the collective efforts of informatics scientists.

\section{References}

1. Romeu J, Sotos F, Ros L, Ortiz A (1995) Health record problem-oriented information system. Medinfo 8: 297.

2. Dolin RH (1995) Modeling the temporal complexities of symptoms. J Am Med Inform Assoc 2: 323-331.

3. George Hripcsak, LZ, Simon Parsons, Amar K Das, Stephen B Johnson (2005) Modeling Electronic Discharge Summaries as a Simple Temporal Constraint Satisfaction Problem. J Am Med Inform Assoc 12: 55-63.

\section{ISSN: 2574-1241}

DOI: 10.26717/BJSTR.2019.19.003326

Min Wu. Biomed J Sci \& Tech Res

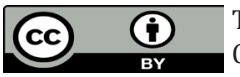

This work is licensed under Creative Commons Attribution 4.0 License

Submission Link: https://biomedres.us/submit-manuscript.php

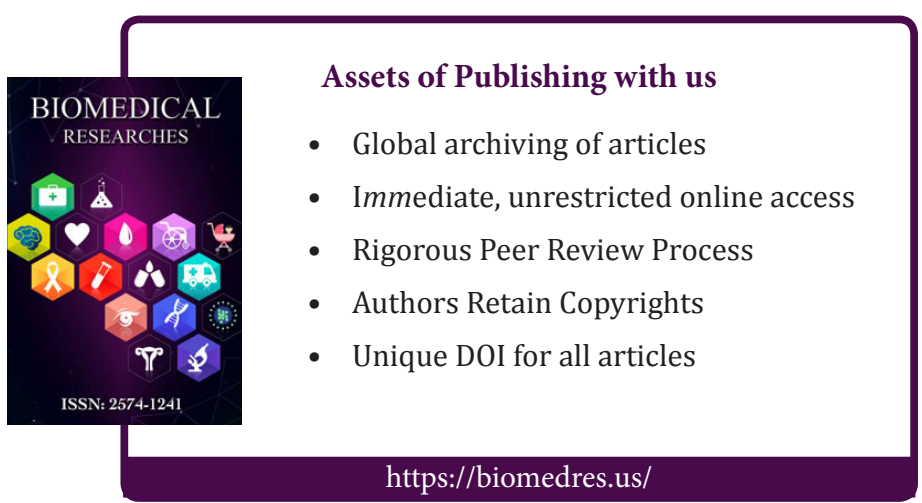

